\title{
26107 - RIGHT VENTRICULAR STRAIN AND STRAIN RATE: TEE VS TTE
}

\section{Claude Tousignant MD, Richard Bowry, MB BS; St. Michael's Hospital, Toronto, ONTARIO, Canada}

INTRODUCTION: The aim of this study was to assess the feasibility of measuring tissue Doppler derived right ventricular (RV) strain (å) and strain rate (SR) in ventilated patients using transesophageal echocardiography (TEE) and to compare with transthoracic echocardiography (TTE).

METHODS: After IRB approval and informed consent, 15 patients undergoing cardiac surgery were enrolled. Under general anesthesia a tissue Doppler color sector was applied to the lateral tricuspid annulus in the apical four chamber view using a GE vivid 7 with $1.7 / 3.4 \mathrm{MHz}$ probe. 1 loop of at least 6 cycles with a frame rate $>200 \mathrm{fps}$ was recorded and stored digitally. This was repeated using a 5.0 MHz multiplane TEE probe in a modified transgastric (TG) RV inflow outflow view (1). The data was analyzed offline using dedicated software. A $6 \times 2 \mathrm{~mm}$ sample was placed in the ventricular wall at the base of the RV (lateral wall for TTE, inferior wall for TEE). The sample was rotated and placed with care to avoid sampling outside the myocardial wall. Measurements of å; and SR were made using 40ms Gaussian smoothing and Baseline å; drift was adjusted by returning to zero after every beat. 4 beats were averaged for each patient per method (TTE and TEE). Student's t test was used to compare the two methods. Linear regression and Bland and Altman plots were used to assess agreement between the two methods.

RESULTS: 15 patients with mean age $63 \pm 10$ years were included. Measurements could not be resolved for å; in 1 patient and SR in 2 patients (excessive noise). Mean å; and SR was $-33.2 \pm 10.9 \%$ and $-2.35 \pm 0.931 / \mathrm{s}$ respectively for TTE and $-34.3 \pm 7.5 \%$ and $2.57 \pm 0.751 / \mathrm{s}$ for TEE. There was no significant difference between the two methods for either å; or SR ( $\mathrm{p}=0.537$ and 0.17 respectively.). When comparing both methods, the $\mathrm{r}$ value was $0.67(\mathrm{p}=0.01)$ for $\mathrm{a}$; and $0.30(\mathrm{p}=0.327)$ for SR. Bland and Altman plots (figure 1) demonstrate a bias for both å; (1a) and SR (1b) toward TEE (-3.8 \% and -0.217 $1 / \mathrm{s}$ respectively) with limits of agreement of $-17 \%$ and $9 \%$ for å; and -2.2 and $1.781 / \mathrm{s}$ for SR

DISCUSSION: å; is a load-dependent measure of regional ventricular function which has been correlated with ejection fraction whereas SR is a more load-independent measure of local contractility $(2,3)$. Because the RV is thin-walled, it is more difficult to measure tissue derived indices of ventricular function. We found that longitudinal å; and SR can be measured in the RV using both TEE and TTE methods. There was a slight bias toward TEE measurements however; the limit of agreement was moderate for å; and very large for SR. Noise remains a significant factor is the assessment of RV function by tissue Doppler imaging. A larger sample size is required to improve the power of this study.

REFERENCES:

1. JASE Jan 2006

2. J Am Soc Echocardiography 2004; 17:788-802

3. Am J Physiol Heart Circ Physiol 2002; 283: H792-99 


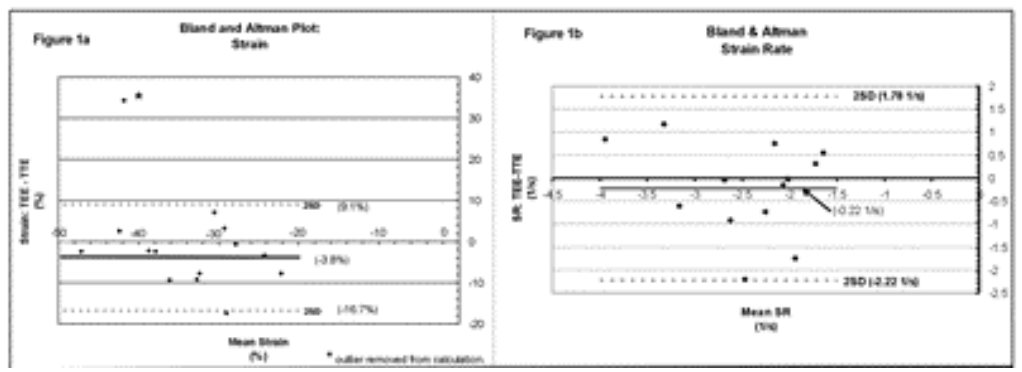

